\title{
Fuzzy Adaptive Modified PSO Algorithm Assisted to Design of Six Order Elliptic Low Pass Filter
}

\author{
Majid Akhlaghi \\ Young Researchers and Elite Club, Omidieh Branch, Islamic Azad University, Omidieh, Iran \\ *Corresponding Author: majidakhlagh@gmail.com
}

Copyright (C) 2014 Horizon Research Publishing All rights reserved.

\begin{abstract}
To provide cost-effective communication systems for separate a signal into different frequency region and eliminate adverse signals and harmonics, Fuzzy Adaptive Modified particle swarm optimization (FAMPSO) method was used to have an optimized gain ripple of $6^{\text {th }}$ order elliptic low pass filter with $300 \mathrm{MHz}$ cut-off frequency. The feasibility and effectiveness of the FAMPSO algorithm is demonstrated and results are compared with PSO algorithm. It is shown that FAMPSO has high quality solution, superior convergence characteristics and shorter computation time.
\end{abstract}

Keywords Optimization Algorithm, Low Pass Filter

\section{Introduction}

Electronic-filter design, whether analog, digital, or distributed, is an essential part of many electrical engineers' workdays [1-6]. Frequency-selective networks are useful for suppressing noise, rejecting unwanted signals, or in some way manipulating the input signal's characteristics. In most communication systems, the low pass filters are used to eliminate adverse signals and harmonics [7]. One of the low pass filters is elliptic filter. An elliptic filter is a signal processing filter with equalized ripple behavior in both the pass band and the stop band $[8,9]$. The following figure 1 clearly has shown the characteristic of the even and odd orders low pass elliptic filter.

As shown, the elliptic filter has an extremely sharp cutoff frequency which makes it ideally suited for filter design cases where there must be severe attenuation in frequencies just entering the stop band of the filter. The amount of the ripple in each band is independently adjustable, and no other filter of equal order can have a faster transition in given between the pass band and the stop band for the given values of ripple. The elliptic filter is an excellent candidate for a low pass filter where the amount of error needs to be minimized on both sides of the cutoff frequency. The amount of ripple is determined by frequency response coefficient. In [10] given tedious and traditional method for determination of these coefficients. In this method probability of minimize error is low and error are compatible than ideal filter. In addition to Gradient-based methods, the Optimization evolutionary optimization algorithms are presented. The advantage of these techniques is it is not require derivative of the cost function to find the global best. Derivative and gradient base algorithms for solving non-convex problems are not capable of making a distinction between local optimal solutions and rigorous optimal solutions, and will treat the former as actual solutions to the original problem. Evolutionary algorithms are capable of guaranteeing convergence in finite time to the actual optimal solution of a non-convex problem is called global optimum. In this paper the filter coefficients are designed using two powerful evolutionary algorithms such as PSO and FAMPSO and compare the results.

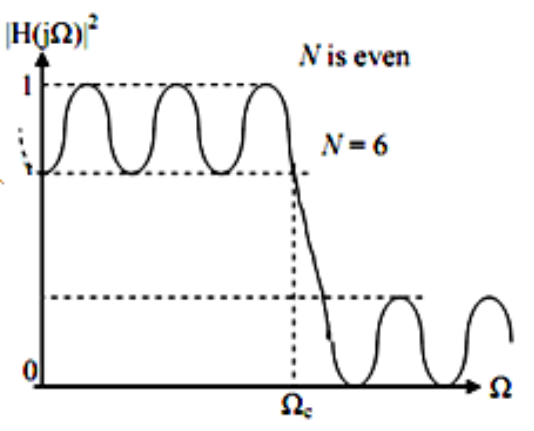

Figure 1. characteristic of the even elliptic Low pass filter

\section{Materials and Methods}

\subsection{Theory}

Frequency response of a $6^{\text {th }}$ order elliptic filter is given by:

$$
H(s)=\frac{a_{1} S^{6}+a_{2} S^{5}+a_{3} S^{4}+a_{4} S^{3}+a_{5} S^{2}+a_{6} S+a_{7}}{b_{1} S^{6}+b_{2} S^{5}+b_{3} S^{4}+b_{4} S^{3}+b_{5} S^{2}+b_{6} S+b_{7}}
$$

As can be seen from Eq. (1), several filter coefficients have to be considered to construct the filter. These 
combinations of variables can affect much on the filter response such as the rise or fall frequency, the ripple at response frequency and the amount of the ripple in decibel (dB). Symbols a1...a7 and b1...b7 denote the filter parameters and the transfer function denoted with $H$. In this paper these coefficients are designed using FAMPSO algorithm to have a low pass filter with minimum ripple in the pass and stop bands.

\subsection{PSO Algorithm}

PSO is an optimization algorithm inspired by a habitation such as birds. This method is based on two points: artificial live and evolution. In the extension of PSO algorithm, the possible solutions of the desired optimization problem are regarded as non-bulky birds and any element of this colony called particle. They can fly in an n-dimensional space and correct its trajectory based on the previous experiments as well as the neighboring particles. For particle number $i$, the displacement vector $\mathbf{X}_{\mathbf{i}}$ is defined as [11]:

$$
\mathbf{X}_{\mathbf{i}}=\left[x_{i 1}, x_{i 2}, \ldots \ldots \ldots, x_{i n}\right]^{T} \in S
$$

Where $S$ is the searching space. This particle consists the velocity vector $\mathbf{V}_{\mathbf{i}}$ in the form of:

$$
\mathbf{V}_{\mathbf{i}}=\left[v_{i 1}, v_{i 2}, \ldots \ldots \ldots, v_{i n}\right]^{T} \in S
$$

The best former position $\mathbf{P}_{\mathbf{i}}$ in the search space of $S$, is defined as:

$$
\mathbf{P}_{\mathbf{i}}=\left[p_{i 1}, p_{i 2}, \ldots \ldots \ldots, p_{\text {in }}\right]^{T}
$$

Using these definitions, we can find the new positions by the aid of some weighting and learning factors which affect the algorithm searching procedure, severely [12].

$$
\begin{gathered}
V_{i}^{k+1}=w V_{i}^{k}+c_{1} r_{1}\left(P_{i}^{k}-X_{i}^{k}\right)+c_{2} r_{2}\left(P_{g}^{k}-X_{i}^{k}\right) \\
X_{i}^{k+1}=X_{i}^{k}+V_{i}^{k+1}
\end{gathered}
$$

Where $V_{i}{ }_{i}$ is velocity of particle $\mathrm{i}$ at iteration $\mathrm{k}, w$ is inertia weight factor, $c_{1}, c_{2}$ are acceleration constants, $x_{i}^{k}$ is the position of particle $\mathrm{i}$ at iteration $\mathrm{k}, P_{i}^{k}$ is the best position of particle i until iteration $\mathrm{k}, P_{g}^{k}$ is the best position of group until iteration $\mathrm{k}$ and finally $\mathrm{r} 1, \mathrm{r} 2$ are random number between 0 and 1 . There are three tuning parameters; $c_{1}, c_{2}$ and $w$ which can control the algorithm behavior severely. We can use $w$ to affect the former velocity on the next velocity. Increasing $w$ causes an increment in searching ability and decreasing $w$ can continue an accurate searching in the previously scanned areas. So, a proper selection provides a compromise between the local and the global searches. In many works, the selected $w$ was big firstly and after an initial search, this value would be lowered; a linearized model. $c_{1}$ and $c_{2}$ are the best private and global positions, respectively. Since $c_{1}$ is the amount of particle trust in the previous experiments, it is called cognitive parameter, whereas $c_{2}$ describes the trust amount on the group and hence it is entitled the social parameter. For $c_{1}>c_{2}$ the particle is attracted to the best private position and the reverse is true for the other case. Many optimization procedures utilize $c_{1}=c_{2}=2$.

\subsection{Fuzzy Formulation for Tuning of W}

Weight coefficient determines the effect of the past velocity experience on the present one. The right choice of this parameter results in the balance between global and local search of PSO algorithm. Great weight coefficient enhances the global search performance and small weight coefficient improves the local search. The parameter $w$ is often held constant or linearly changed for the entire run of a PSO but this method cannot obtain the global optima in many cases. When the environment itself is dynamically changed over the time, the optimization algorithm should be able to adapt dynamically to the changing environment. The change of the particle's situation is directly correlated to the inertia weight. The best choice for adjusting the computed weight coefficient is based on changes in G_best_value and in this regard fuzzy logic method is applied. The inputs of fuzzy method are NFV and $w$ while the output of this method is $\Delta w$ which are computed as follows [13]:

$$
N F V=\frac{\left(F V-F V_{\min }\right)}{\left(F V_{\max }-F V_{\min }\right)}
$$

In Eq.(7) $\mathrm{FV}$ is a current best performance evaluation and the calculated value of FV from Eq. 13 at the first iteration may be used as $F V_{\min }$ for the next iterations. $F V_{\max }$ is a very large value which is greater than any feasible solution. Usual inertia weight value is about $0 / 4<w<0 / 9$. In the fuzzy set, both positive and negative corrections are essential for the inertia weight. Therefore, for the inertia weight correction, a range of _ 0.1 to 0.1 has been selected [13].

$$
w^{k+1}=w^{k}+\Delta w
$$

For achieving the optimal point, objective functions should be described by membership functions. In this paper, for simplicity, all the membership functions are modeled with triangular shape and also they can take one of the three following status: Small (S), Medium (M) and Large (L). In Table 1, output variables of fuzzy sets are presented in three forms of linguistic values; NE (Negative), ZE (Zero), and PE (Positive) with related membership functions, as shown in Fig.2. Rules of fuzzy sets are shown in Table 1. There are nine possible rules for two input variables and three linguistic values for all input variables. Each fuzzy rule as from as "IF-THEN" statement like the following rule, for example: If $N F V$ is $(L)$ and $w$ is $(M)$ then $\Delta w$ is $(Z E)$. The fuzzy rules are shown in Table 1 used to modify the inertia weight correction $(\Delta w)$. Each rule represents a relation between the input and output spaces. The membership functions are depicted in Fig. 2 are utilized for fuzzified each input and output variable [13].

\subsection{Modified PSO}

One of the disadvantages of the original PSO is falling in a local optimum point, so in order to improve the convergence 
property and accuracy of the PSO algorithm, this paper presented a modified particle swarm optimization method. Premature convergence can take place under different situations such as the population has converged to local optima, the population has lost its diversity, and the search algorithm has proceeded slowly or hasn't proceeded at all. Mutation is a powerful strategy to increase the population diversity and improve the PSOs performance. This paper employed a new mutation operator described as follows: In each iteration, three vectors are selected randomly $(Z 1, Z 2$, Z3) from the initial population after these vectors are mutated. It is worthwhile noting that for seeking the search space uniformly, the selected vectors should be different $(Z 1 \neq Z 2 \neq Z 3)$. A mutant vector $(X m u t)$ is generated as [14]:

$$
\mathbf{X}_{m u t}=\mathbf{X}_{z 1}+\beta^{*}\left(\mathbf{x}_{z 2}-\mathbf{X}_{z 3}\right)
$$

Where $]^{*}$ is the mutation constant with the value of: $0.93<\theta^{*}<1$. The target vector is mixed with the mutated vector, using the following scheme to yield the trial vector:

$$
\mathbf{X}_{\text {new }, i}=\left\{\begin{array}{cc}
\mathbf{x}_{\text {mut }, i}, & \text { if }(\text { rand }<\text { crossover }) \\
\mathbf{x}_{i}, & \text { Otherwise }
\end{array} \quad i=1,2, \ldots \ldots ., n\right.
$$

Usual crossover is selected in the range of: $0.1<$ crossover $<0.9$. In this paper $\beta^{*}=0.93$ and crossover $=$ $0.1[14]$.
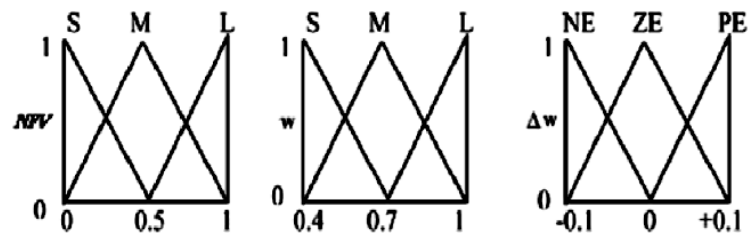

Figure 2. The membership functions

\begin{tabular}{|c|c|c|c|c|}
\hline \multirow{2}{*}{\multicolumn{2}{|c|}{$\Delta w$}} & \multicolumn{3}{|c|}{ w } \\
\hline & & $\mathrm{S}$ & $\mathrm{M}$ & $\mathrm{L}$ \\
\hline \multirow{3}{*}{ NFV } & $\mathrm{S}$ & $\mathrm{ZE}$ & $\mathrm{NE}$ & NE \\
\hline & $\mathrm{M}$ & PE & ZE & $\mathrm{NE}$ \\
\hline & L & PE & ZE & $\mathrm{NE}$ \\
\hline
\end{tabular}

Table 1. Fuzzy Rules of the Input and Output Variables

\subsection{Adaptive PSO}

In the case of large global minimum the particles are far away from the optimum point. Hence to search globally the solution space, a big velocity is needed. Therefore, $c_{l}$ and $c_{2}$ must be larger values. Conversely, only small movements and $c_{1}$ and $c_{2}$ is required and must be chosen to small values. In this paper, during search process of PSO algorithm, learning factors are set as a function of global optimum ripple. In this way, the acceleration coefficients are proposed as follows [15]:

$$
c_{1,2}=1+\left[1+\exp \left(-\frac{G_{-} \text {best_value }}{G_{0}}\right)^{n}\right]^{-1}
$$

We chose $\mathrm{n}=2$ and $G_{0}$ as the $G$ best_value in the first iteration.

\section{Numerical Result}

Consider an ideal low pass filter with $300 \mathrm{MHz}$ cut-off frequency. Our goal is to minimize the equalized ripple behavior in both the pass band and the stop band by utilize of FAMPSO method to introduce optimizing fourteen parameters; with the following intervals:

$$
\begin{aligned}
& a_{1}-a_{7} \in\left[\begin{array}{ll}
-2 & 2
\end{array}\right] \\
& b_{1}-b_{7} \in\left[\begin{array}{ll}
-2 & 2
\end{array}\right]
\end{aligned}
$$

Such minimization must be done around a predefined ideal filter. In other words, this procedure should be minimized by the following function:

$$
\text { Fitness function }=\operatorname{MSE}(\mathrm{e}), \mathrm{e}=\mathrm{G}-\bar{G}
$$

In this equation MSE is mean squared error and $\bar{G}$ is predefined ideal filter which shown in Fig. 3 and minimization must be done around it.

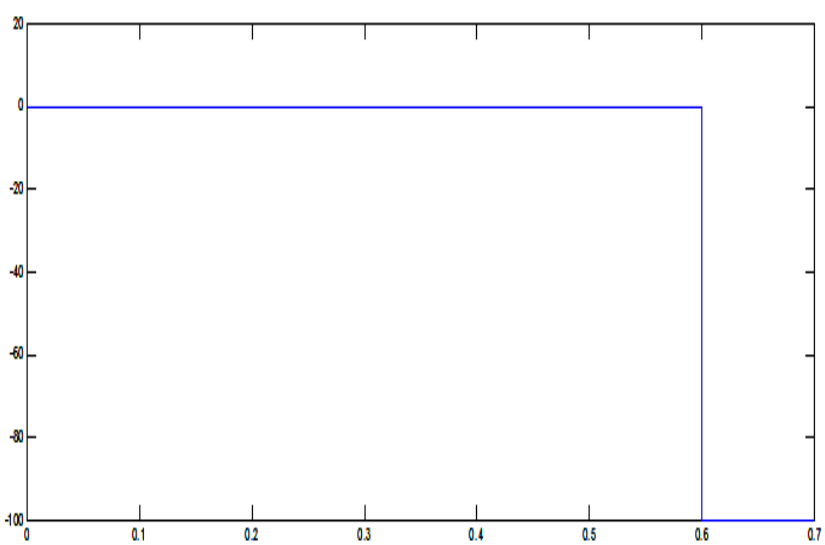

Figure 3. Ideal Low Pass Filter

Application of the PSO algorithm to optimize the equalized ripple of an elliptic filter is plotted in fig. 4 for a filter with $300 \mathrm{MHz}$ cut of frequency. In this case, MSE coefficient of the Frequency response around ideal filter restricted to a range of about 1484. Our efficient FAMPSO algorithm is proposed to minimize the gain ripple of $6^{\text {th }}$ order elliptic low pass filter with $300 \mathrm{MHz}$ cut-off frequency. In our simulations, the number of particles is considered to be three times of the optimization parameters $(3 \times 14$ parameters). The increase of the number of particles can improve the convergence speed initially, whereas it will be reduced the accuracy and convergence speed for more iterations. As shown in Fig. 5, FAMPSO is much more powerful than PSO algorithm (note that PSO algorithm is usually trapped in some local minimum).The optimization results for filter coefficients are shown in Table 2.

The convergence characteristic of the proposed method is 
compared with that of PSO and the results are shown in Fig. 6. Indeed, FAMPSO algorithm is performing better than PSO in terms of convergence speed and accuracy. Although the quality of proposed algorithm is improved with compare with the original PSO algorithm, the consumption time for running the algorithm increased.

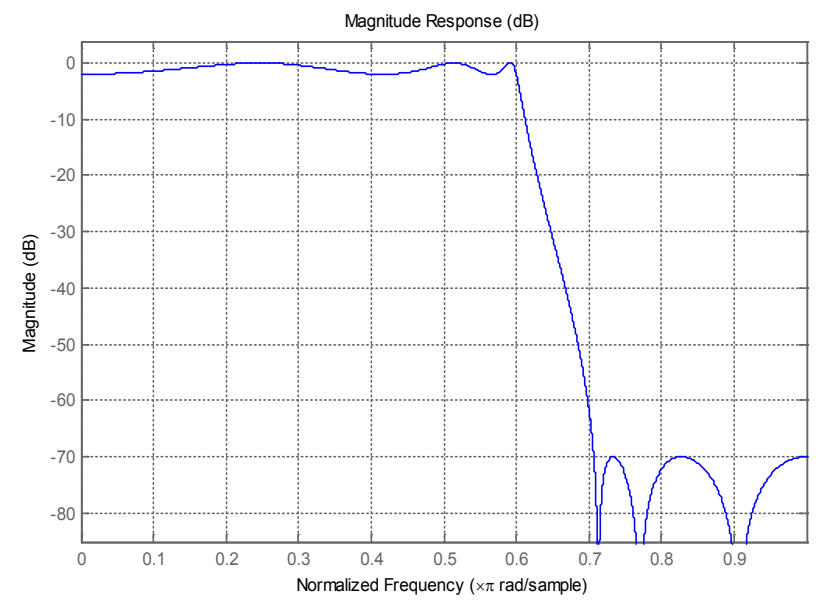

Figure 4. filter Characteristic which optimized by PSO algorithm

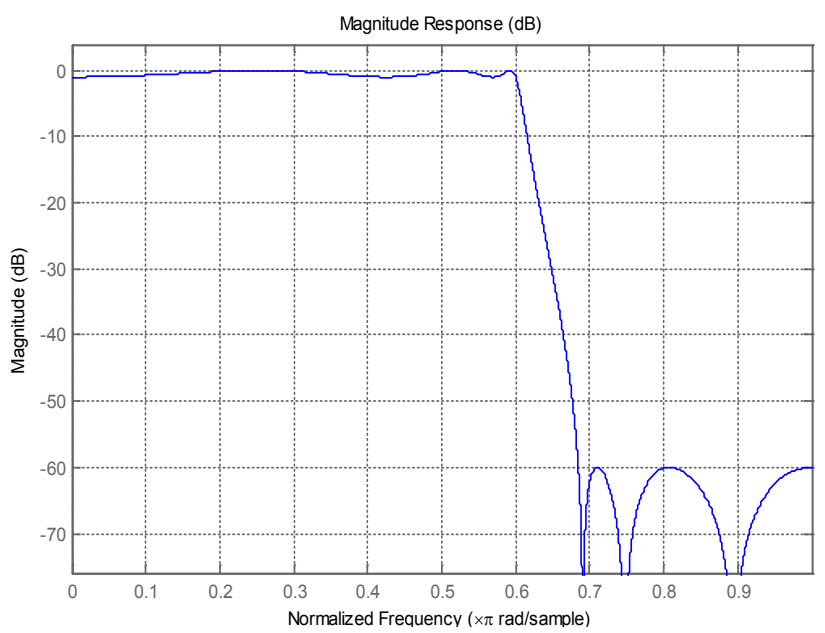

Figure 5. filter Characteristic which optimized by FAMPSO algorithm

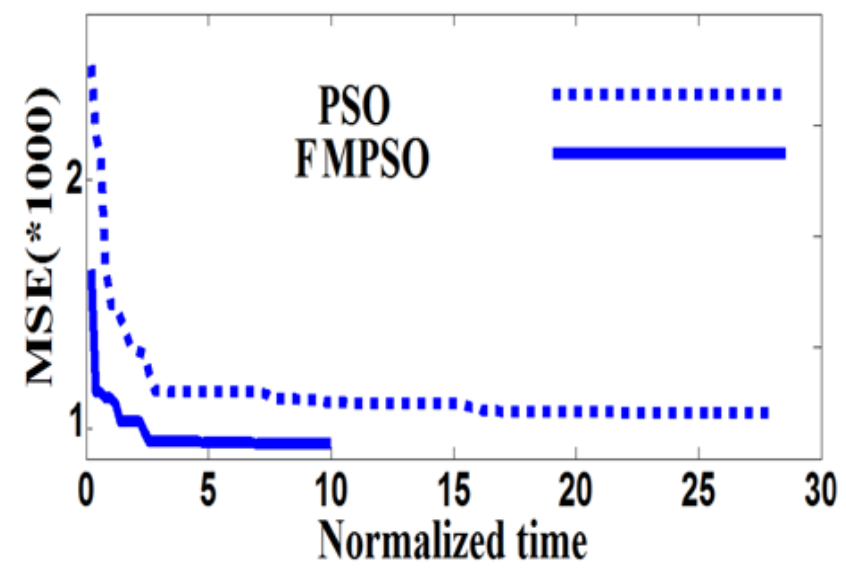

Figure 6. MSE versus the number of iteration for the standard PSO and the proposed FAMPSO
Table 2. Optimization Results for Six order Elliptic Filter

\begin{tabular}{|c|ccccc|}
\hline & 0.0474 & 0.2208 & 0.4795 & 0.6107 & 0.4795 \\
& 0.2208 & 0.0474 & 1.000 & -0.1205 & 1.558 \\
& -0.6255 & 0.9254 & -0.3594 & 0.2734 & \\
\hline \multirow{3}{*}{ FAMPSO } & 0.0674 & 0.298 & 0.6314 & 0.7977 & 0.6314 \\
& 0.298 & 0.0674 & 1.000 & 0.1614 & 1.5266 \\
& -0.271 & 0.7655 & -0.2338 & 0.1833 & \\
\hline
\end{tabular}

\section{Conclusion}

An optimized algorithm based on PSO optimization method was proposed to find a minimum gain ripple for $6^{\text {th }}$ order elliptic low pass filter. To find the global answer instead of the local minima, we utilized a combinational algorithm called FAMPSO for optimizing the gain ripple and found the proper frequency response elliptic filter coefficients. The proposed method was applied by a six order elliptic low pass filter. It was found that the proposed algorithm can give fewer ripples and hence uniform gain in the related bandwidth with less iterations and higher accuracy.

\section{REFERENCES}

[1] X. P. Lai , Z. P. Lin and H. K. Kwan "A sequential minimization procedure for minimax design of IIR filters based on second-order factor updates", IEEE Trans. Circuits Syst. II, Exp. Briefs, vol. 58, no. 1, pp.51 -55 2011

[2] X. P. Lai "Optimal design of nonlinear-phase FIR filters with prescribed phase error", IEEE Trans. Signal Process., vol. 57, no. 9 , pp.3399 -3410 2009

[3] A. Jiang and H. K. Kwan "Minimax design of IIR digital filters using iterative SOCP", IEEE Trans. Circuits Syst. I, Reg. Papers, vol. 57, no. 6, pp.1326 -1337 2010

[4] J. Pekonen, J. Nam , J. Smith and V. V\&auml;lim\&auml;ki "Optimized polynomial spline basis function design for quasi-bandlimited classical waveform synthesis", IEEE Signal Process. Lett., vol. 19, no. 3, pp.159-162 2012

[5] R. M. Joshi , A. Madanayake , L. T. Bruton and M. Maini "Discrete-space continuous-time analog circuits for spatially-bandpass 2D IIR beam filters", Proc. Int. Workshop Multidimensional nDS, pp.1 -7 2011

[6] H. Dong, Z. Wang and H. Gao "Distributed filtering for a class of time - varying system over sensor networks with quantization error and successive packet dropouts", IEEE Trans. Signal Process., vol. 60, no. 6, pp.3164-3173 2012

[7] Sheen, Jyh-Wen "A compact semi-lumped low-pass filter for harmonics and spurious suppression" Microwave and Guided Wave Letters, IEEE, Volume: 10 , Issue: 3 , 2000 , Page(s): $92-93$

[8] J. A.G, Malherbe "Pseudo- elliptic bandpass filter with subharmonic stubs" Electronics Letters, vol. 47, no. 3, 
pp.196-198 (2011)

[9] Malherbe, J.A.G. ; Reid, C.A. "Double resonant stub bandstop filter with pseudo-elliptic response"Electronics Letters Vol.46, no. 7, 2010 , Page(s): 508 - 509

[10] S. J. Orfanidis, "Lecture Notes on Elliptic Filter Design", Department of Electrical \& Computer Engineering, Rutgers University, 2006.

[11] T. Niknam, H. Mojarrad, M. Nayeripour, "A new fuzzy adaptive particle swarm optimization for non-convex economic dispatch," Int. J. Innovative Computing, Information and Control. 7, 1764-1778 (2011).

[12] T. Niknam, H. D. Mojarrad, and H.Z Meymand, "A novel hybrid particle swarm optimization for economic dispatch with valve-point loading effects," Applied Energy Conversion and Management. 52, 1800-1809 (2011).

[13] P. Bajpai, and S. N. Singh, "Fuzzy adaptive particle swarm optimization for biddinrg strategy in uniform price spot market," IEEE Trans. Power Sys. 22, 2152-2160 (2007).

[14] F. Emami, M. Akhlaghi, "Gain ripple decrement of S-band Raman amplifier." J. Photonics Technology letters. 24, 1349-1352 (2012).

[15] M. Akhlaghi and F. Emami, "Fuzzy Adaptive Modified PSO-Algorithm Assisted to Design of Photonic Crystal Fiber Raman Amplifier," J. Opt. Soc. Korea 17, 237-241 (2013) 